\title{
A new Cryptographic Algorithm AEDS (Advanced Encryption and Decryption Standard) for data security
}

\author{
Ali Mohammed Ali Argabi ${ }^{1}$, Md Imran Alam ${ }^{2}$ \\ Student, Department of Computer and Network Engineering, College of CS \& IT, Jazan, Saudi Arabia ${ }^{1}$ \\ Lecturer, Department of Computer and Network Engineering, College of CS \& IT, Jazan, India ${ }^{2}$
}

\begin{abstract}
Nowadays security is very important to protect our sensitive information in computer or over the internet such as in online banking, online shopping, stock market and bill payments etc. Without security our information exchanged over internet are not safe. Encryption Algorithms provides the security to the information which is exchanged over the internet. In this project we are proposing a new cryptographic algorithm AEDS (Advanced Encryption and Decryption Standard) which is developed by combining properties of DES and AES algorithms. Then we compared all these three algorithms and we found that AEDS is more secure and robust for data security.
\end{abstract}

Keywords: Encryption, Algorithms, AEDS, DES, AES, Security, Internet

\section{INTRODUCTION}

Nowadays security is very important to protect our sensitive information in computer or over the internet such as in online banking, online shopping, stock market and bill payments etc. Without security our information exchanged over internet are not safe. Encryption Algorithms provides the security to the information which is exchanged over the internet. Encryption algorithms play a big role in providing data security against malicious attacks. There are two types of Encryption Algorithms:

First type is Symmetric key encryption also called private key or one key encryption algorithm. In this algorithm same key is used to encrypt and decrypt data, such as DES (Data Encryption Algorithm) and AES (Advanced Encryption Standard) algorithms.

Second type is Asymmetric key also called public key encryption algorithm, which uses one key for encryptions of data and other key for decryption of data such as RSA and ElGamal algorithms.

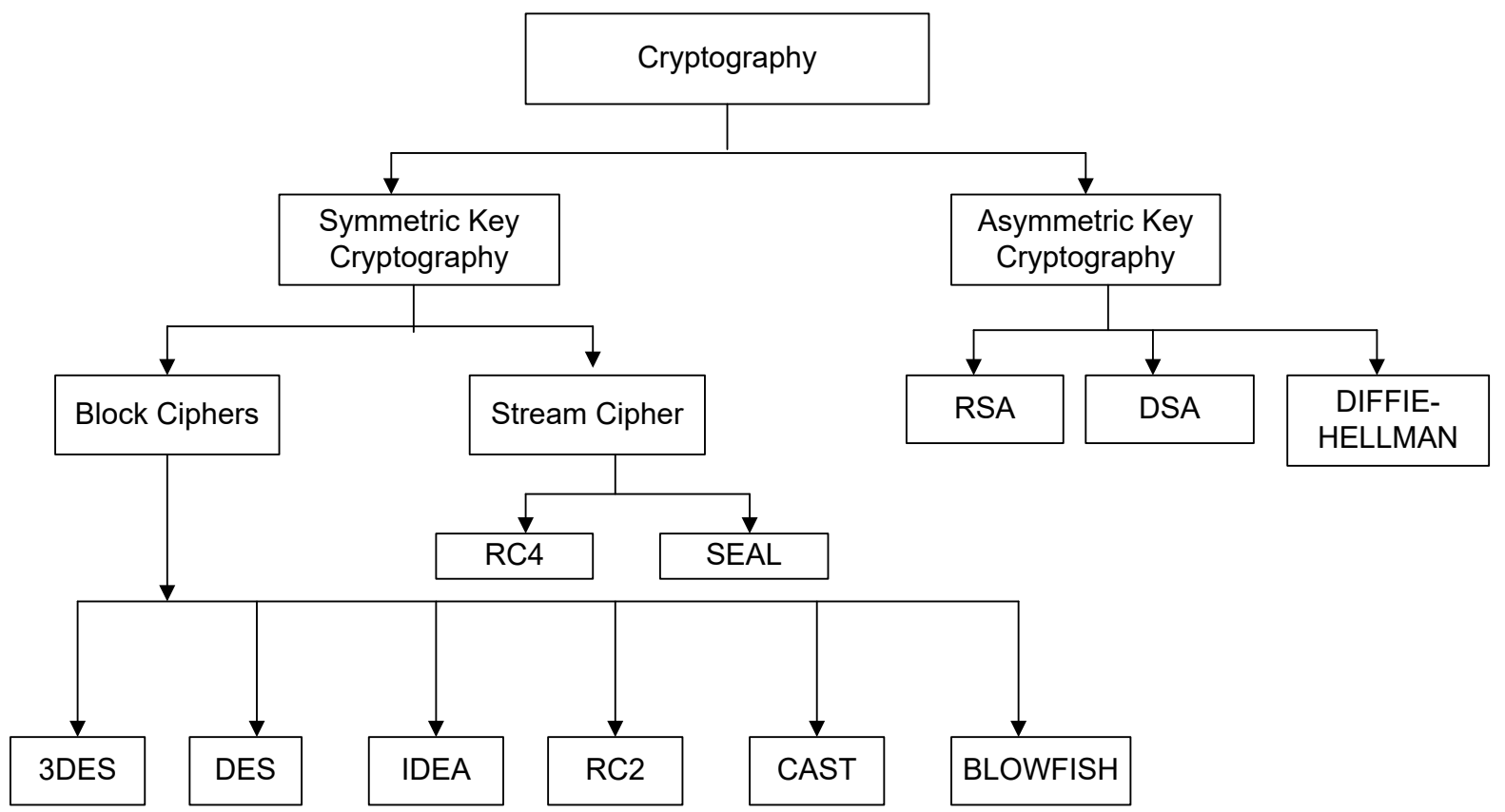

Fig. 1 


\section{International Advanced Research Journal in Science, Engineering and Technology}

Vol. 6, Issue 10, October 2019

Above Fig. 1 shows classification and some invented algorithms of cryptography.

In this paper we are proposing a new cryptographic algorithm AEDS (Advanced Encryption and Decryption Standard) which is developed by combining DES and AES algorithms. We expect AEDS will be more secure and robust as compared to DES and AES.

\section{LITERATURE REVIEW}

In this section, the various performance factors and techniques used for encrypting and decrypting data used by various research papers are listed.

In the research paper [1] various experimental factors are analysed. Based on the text files used and the experimental results it was concluded that DES algorithm consumes least encryption time and AES algorithm use least memory usage. Encryption time differs in case of AES algorithm and DES algorithm.

Paper[2] presents a performance evaluation of selected symmetric encryption algorithms. The selected algorithms are AES, DES, and 3DES, RC6, Blowfish and RC2. In the case of changing data type such as image instead of text, it was found that RC2, RC6 and Blowfish has disadvantage over other algorithms in terms of time consumption.

In the research paper [3] shown a new comparative study between encrypting techniques were presented in to nine factors, which are key length, cipher type, block size, developed, cryptanalysis resistance, security, possibility key, possible ACSII printable character keys, time required to 170 check all possible key at 50 billion second, these eligible proved the AES is better.

Paper [4] surveyed the existing encryption techniques like AES, DES and RSA algorithms along with LSB substitution technique. Those encryption techniques are studied and analysed well to promote the performance of the encryption methods also to ensure the security. Based on the experimental result it was concluded that AES algorithm consumes least encryption and decryption time and buffer usage compared to DES algorithm, but RSA consume more encryption time and buffer usage is also very high. We also observed that decryption of AES algorithm is better than other algorithms. From the simulation result, we evaluated that AES algorithm is much better than DES and RSA algorithm

In Paper [5] it is discussed that in symmetric key encryption techniques the AES algorithm is specified as the better solution then follows the blowfish algorithm. In the Asymmetric encryption technique, the RSA algorithm is more secure key generation. since it uses the factoring of high prime number hence, the RSA algorithm is found as the better solution in this method.

\section{OBJECTIVES AND IMPORTANCE OF RESEARCH TO SOCIETY}

\section{Our Objectives are as follows:}

We implemented DES, AES and new Cryptographic algorithm AEDS in Java programming language. Then we compared these algorithms based on parameters like encryption time and decryption time. The encryption time is considered the time that an encryption algorithm takes to produce a cipher text from a plaintext. The decryption time is considered the time that a decryption algorithm takes to reproduce a Plaintext from a Cipher text.

Based on decryption time, we analysed our algorithm is stronger than other two algorithms (AES and DES) or not. Our algorithm AEDS is taking more time in decryption as compared to previous algorithms AES and DES, it means our algorithm is more complex and not easy to break. So, we can say our proposed algorithm is stronger than others.

\section{Importance of research to society}

Nowadays Computers have a huge impact on society and the world will never be again the way it was before computers. Research in one of the field of computer science (Cryptography) has positive impact on humankind and society in more ways than we can think of.

Below are few of the importance of research to society in Cryptography field of Information security. Security of data is very important for most online businesses and even home computer users. Client information, online payment 


\section{International Advanced Research Journal in Science, Engineering and Technology}

Vol. 6, Issue 10, October 2019

transactions, personal files, bank account details - all of this information can be hard to replace and potentially dangerous if it falls into the wrong hands. Data lost due to disasters such as a flood or fire is crushing, but loosing data in to wrong hands of hackers or a malware infection can have much dangerous impact on humankind and society. To prevent data loss and protecting information of user's cryptographic techniques are used.

\section{RESEARCH METHODOLOGY}

The experiment is performed on three different machines having three different operating systems (Linux, Mac OS X and Windows). We have taken different strings and files as plaintext. Then after executing our algorithms we got different cipher texts. Now we collected encryption time and decryption time of each plaintext from different operating systems. Based on decryption time we decided which algorithm is stronger.

\section{Results:}

Encryption time are shown in the following Tables, When we are taking String( as Plaintext):

\begin{tabular}{|c|c|c|c|c|c|}
\hline \multicolumn{6}{|c|}{ Encryption } \\
\hline Input String & Algorithm & Windows & Mac & Linux & Average \\
\hline \multirow{3}{*}{ ALI } & DES & 41216 & 350932 & 96753 & 162967 \\
\hline & AES & 92160 & 598213 & 191596 & 293989.667 \\
\hline & AEDS & 35072 & 418508 & 92319 & 181966.333 \\
\hline \multirow{3}{*}{ ali } & DES & 30976 & 113396 & 68084 & 70818.6667 \\
\hline & AES & 41216 & 357075 & 85701 & 161330.667 \\
\hline & AEDS & 43520 & 263558 & 88792 & 131956.667 \\
\hline \multirow{3}{*}{ Mohammed } & DES & 35328 & 168712 & 80708 & 94916 \\
\hline & AES & 42752 & 147113 & 155148 & 115004.333 \\
\hline & AEDS & 2699007 & 132912 & 84820 & 972246.333 \\
\hline \multirow{3}{*}{ МАККАН } & DES & 30208 & 113721 & 65614 & 69847.6667 \\
\hline & AES & 40704 & 179890 & 83025 & 101206.333 \\
\hline & AEDS & 224256 & 741971 & 356208 & 440811.667 \\
\hline \multirow{3}{*}{ JAZAN } & DES & 30464 & 130738 & 63554 & 74918.6667 \\
\hline & AES & 38400 & 223771 & 80538 & 114236.333 \\
\hline & AEDS & 33792 & 254879 & 71207 & 119959.333 \\
\hline \multirow{3}{*}{ University } & DES & 64256 & 518721 & 77884 & 220287 \\
\hline & AES & 62208 & 1361121 & 78648 & 500659 \\
\hline & AEDS & 44032 & 109877 & 98536 & 84148.3333 \\
\hline \multirow{3}{*}{ 123@\#\$ } & DES & 27904 & 114656 & 58503 & 67021 \\
\hline & AES & 64256 & 247062 & 87209 & 132842.333 \\
\hline & AEDS & 32512 & 84403 & 68339 & 61751.3333 \\
\hline \multirow{3}{*}{ Jazan123\# } & DES & 32256 & 90671 & 67828 & 63585 \\
\hline & AES & 38656 & 114155 & 79719 & 77510 \\
\hline & AEDS & 55040 & 84607 & 75135 & 71594 \\
\hline
\end{tabular}

\begin{tabular}{|c|c|c|c|c|c|c|c|c|c|}
\hline Encryption & ALI & ali & Mohammed & MAKKAH & JAZAN & University & 123@\#\$ & \begin{tabular}{|l|}
$J a z a n 123 \#$ \\
\end{tabular} & Average \\
\hline DES & 162967 & 70818.6667 & 94916 & 69847.6667 & 74918.6667 & 220287 & 67021 & 63585 & 103045.125 \\
\hline AES & 293989.667 & 161330.667 & 115004.333 & 101206.333 & 114236.333 & 500659 & 132842.333 & 77510 & 187097.333 \\
\hline AEDS & 181966.333 & 131956.667 & 972246.333 & 440811.667 & 119959.333 & 84148.3333 & 61751.3333 & 71594 & 258054.25 \\
\hline
\end{tabular}




\section{International Advanced Research Journal in Science, Engineering and Technology}

Vol. 6, Issue 10, October 2019

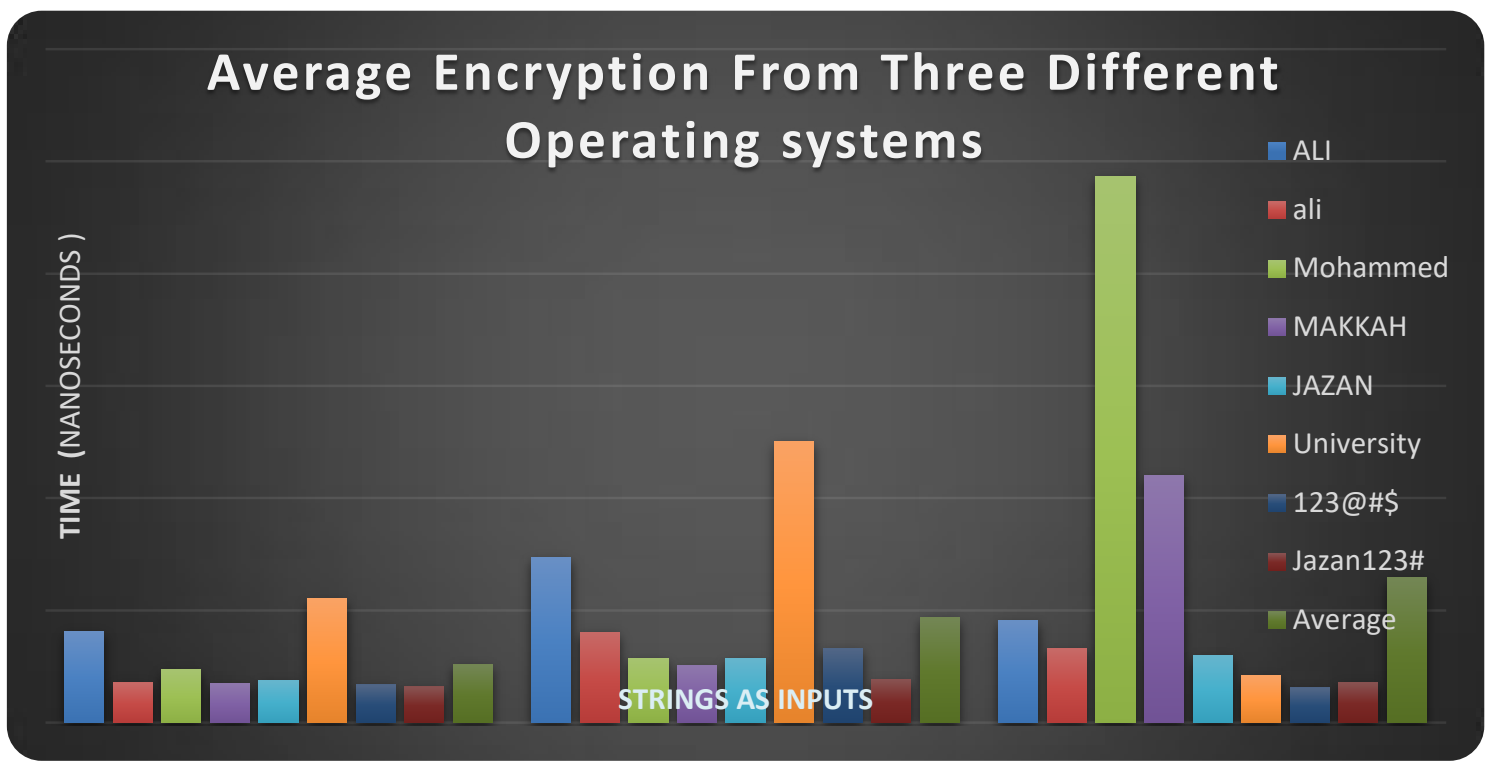

Decryption time are shown in the following Tables, When we are taking String( as Plaintext):

\begin{tabular}{|c|c|c|c|c|c|}
\hline \multicolumn{6}{|c|}{ Decryption } \\
\hline Input String & Algorithm & Windows & Mac & Linux & Average \\
\hline \multirow{3}{*}{ ALI } & DES & 35584 & 258520 & 79401 & 124501.667 \\
\hline & AES & 36096 & 261233 & 80948 & 126092.333 \\
\hline & AEDS & 65792 & 496669 & 146482 & 236314.333 \\
\hline \multirow{3}{*}{ ali } & DES & 39424 & 191548 & 68013 & 99661.6667 \\
\hline & AES & 40960 & 325149 & 78204 & 148104.333 \\
\hline & AEDS & 85504 & 598847 & 196422 & 293591 \\
\hline \multirow{3}{*}{ Mohammed } & DES & 48896 & 107491 & 74885 & 77090.6667 \\
\hline & AES & 42496 & 189938 & 93654 & 108696 \\
\hline & AEDS & 151808 & 321979 & 157266 & 210351 \\
\hline \multirow{3}{*}{ МАKКАН } & DES & 50688 & 112078 & 93080 & 85282 \\
\hline & AES & 47360 & 124553 & 137970 & 103294.333 \\
\hline & AEDS & 77568 & 278649 & 152206 & 169474.333 \\
\hline \multirow{3}{*}{ JAZAN } & DES & 208896 & 698935 & 329612 & 412481 \\
\hline & AES & 38400 & 210030 & 76452 & 108294 \\
\hline & AEDS & 65280 & 270105 & 137273 & 157552.667 \\
\hline \multirow{3}{*}{ University } & DES & 48896 & 262395 & 78765 & 130018.667 \\
\hline & AES & 42496 & 444637 & 72638 & 186590.333 \\
\hline & AEDS & 70656 & 246049 & 143133 & 153279.333 \\
\hline \multirow{3}{*}{ 123@\#\$ } & DES & 27392 & 124945 & 56764 & 69700.3333 \\
\hline & AES & 33280 & 89035 & 70240 & 64185 \\
\hline & AEDS & 59392 & 205009 & 154743 & 139714.667 \\
\hline \multirow{3}{*}{ Jazan123\# } & DES & 31232 & 94187 & 66202 & 63873.6667 \\
\hline & AES & 32768 & 81456 & 68877 & 61033.6667 \\
\hline & AEDS & 64512 & 232186 & 140112 & 145603.333 \\
\hline
\end{tabular}

\begin{tabular}{||c||c||c|c|c|c|c||c||c||c||}
\hline \hline Decryption & ALI & ali & Mohammed & MAKKAH & JAZAN & University & 123@\#\$ & Jaran123\# & Average \\
\hline \hline DES & 124501.667 & 99661.6667 & 77090.6667 & 85282 & 412481 & 130018.667 & 69700.3333 & 63873.6667 & 132826.208 \\
\hline \hline AES & 126092.333 & 148104.333 & 108696 & 103294.333 & 108294 & 186590.333 & 64185 & 61033.6667 & 113286.25 \\
\hline \hline AEDS & 236314.333 & 293591 & 210351 & 169474.333 & 157552.667 & 153279.333 & 139714.667 & 145603.333 & 188235.083 \\
\hline
\end{tabular}




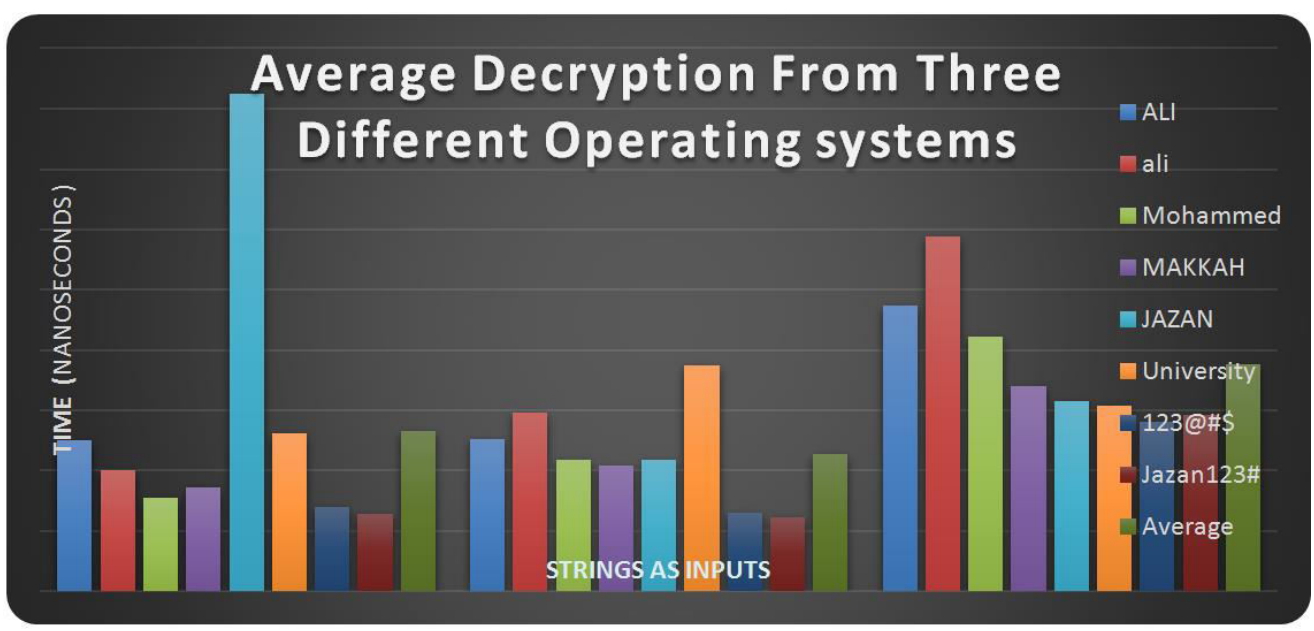

When we are taking Different size of Files (as Plaintext)

Encryption time are shown in the following Tables, When we are taking Different size of Files (as Plaintext):

\begin{tabular}{||c|c|c|c|c|c|}
\hline \multicolumn{7}{|c|}{ Encryption } \\
\hline \hline File Size(Byets) & Algorithm & Windows & Mac & Linux & Average \\
\hline \hline \multirow{3}{*}{8 Bytes } & DES & 54016 & 153407 & 103467 & 103630 \\
\cline { 2 - 6 } & AES & 65280 & 300056 & 101536 & 155624 \\
\cline { 2 - 6 } & AEDS & 100096 & 1710835 & 179786 & 663572.3333 \\
\hline \hline \multirow{3}{*}{35 Bytes } & DES & 200192 & 82907 & 143125 & 142074.6667 \\
\cline { 2 - 6 } & AES & 106496 & 108355 & 120901 & 111917.3333 \\
\cline { 2 - 6 } & AEDS & 201728 & 213411 & 253690 & 222943 \\
\hline \hline \multirow{3}{*}{64 Bytes } & DES & 97024 & 151255 & 157054 & 135111 \\
\cline { 2 - 6 } & AES & 102656 & 163278 & 109562 & 125165.3333 \\
\cline { 2 - 6 } & AEDS & 136192 & 1170621 & 252310 & 519707.6667 \\
\hline
\end{tabular}

\begin{tabular}{||c||c||c|c||c||}
\hline \hline Encryption & 8 Bytes & 35 Bytes & 64 Bytes & Average \\
\hline \hline DES & 103630 & 142074.67 & 135111 & 126938.5556 \\
\hline \hline AES & 155624 & 111917.33 & 125165.333 & 130902.2222 \\
\hline \hline AEDS & 663572.3333 & 222943 & 519707.667 & 468741 \\
\hline
\end{tabular}

\section{Average Encryption From Three Different Operating systems}
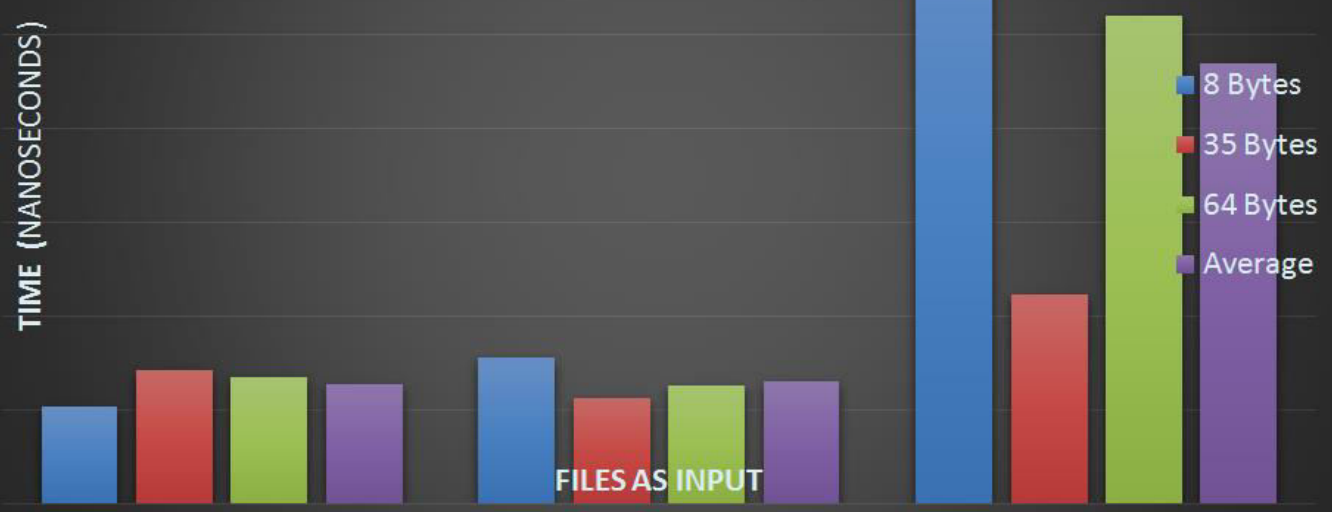


\section{International Advanced Research Journal in Science, Engineering and Technology}

Vol. 6, Issue 10, October 2019

Decryption time are shown in the following Tables, When we are taking Different size of Files (as Plaintext):

\begin{tabular}{||c|c|c|c|c|c|}
\hline \multicolumn{7}{|c|}{ Decryption } \\
\hline \hline File Size(Byets) & Algorithm & Windows & Mac & Linux & Average \\
\hline \hline \multirow{3}{*}{8 Bytes } & DES & 61952 & 231783 & 109350 & 134362 \\
\cline { 2 - 6 } & AES & 56320 & 128061 & 97235 & 93872 \\
\cline { 2 - 6 } & AEDS & 106752 & 199489 & 176471 & 160904 \\
\hline \hline \multirow{3}{*}{35 Bytes } & DES & 117248 & 82405 & 120296 & 106650 \\
\cline { 2 - 6 } & AES & 92928 & 303641 & 98452 & 165007 \\
\cline { 2 - 6 } & AEDS & 150528 & 1586166 & 288406 & 675033 \\
\hline \hline \multirow{3}{*}{64 Bytes } & DES & 66816 & 165995 & 155639 & 129483 \\
\cline { 2 - 6 } & AES & 75264 & 135931 & 107229 & 106141 \\
\cline { 2 - 6 } & AEDS & 146944 & 197996 & 272458 & 205799 \\
\hline \hline
\end{tabular}

\begin{tabular}{||c||c||c|c||c||}
\hline \hline Decryption & 8 Bytes & 35 Bytes & 64 Bytes & Average \\
\hline \hline DES & 134361.67 & 106649.7 & 129483 & 123498.2222 \\
\hline \hline AES & 93872 & 165007 & 106141 & 121673.4444 \\
\hline \hline AEDS & 160904 & 675033.3 & 205799 & 347245.5556 \\
\hline
\end{tabular}

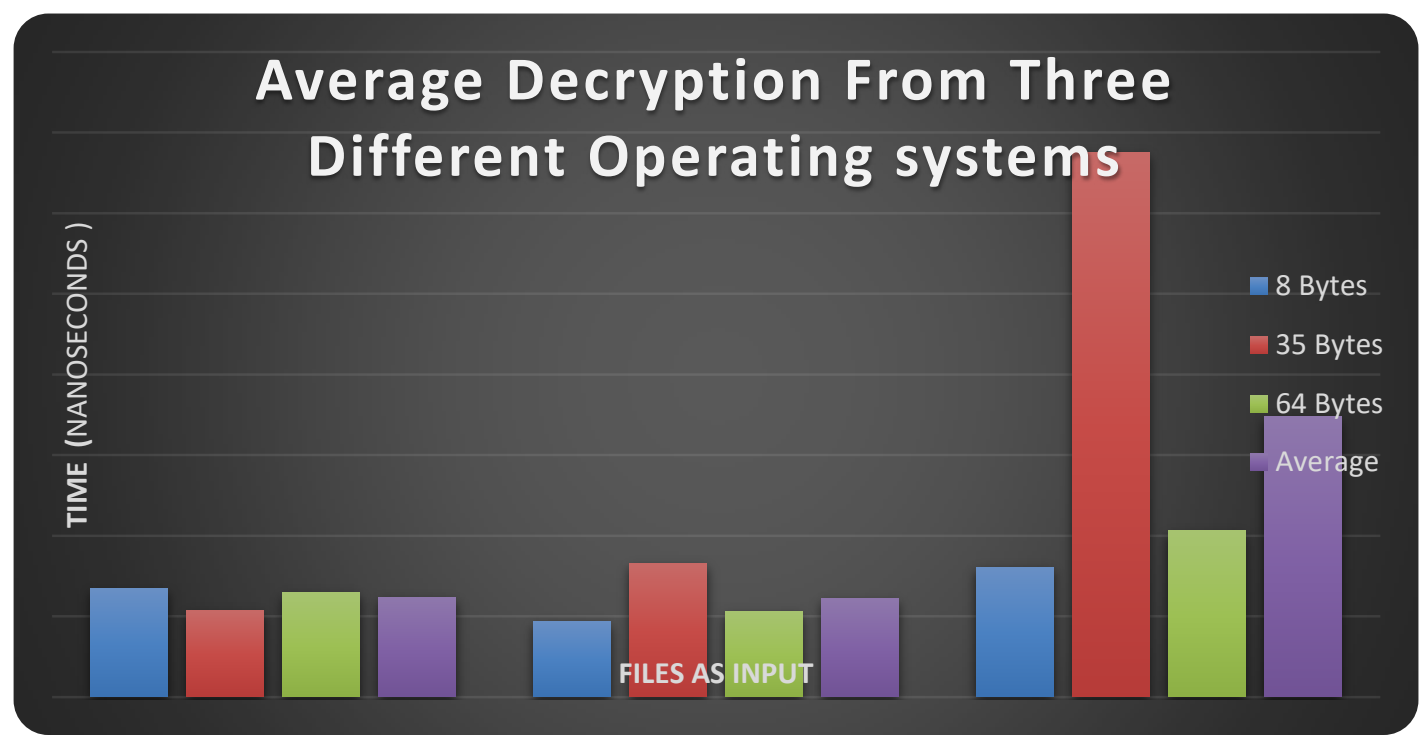

\section{CONCLUSION}

Here we combined the concept of DES and AES Algorithms and developed a new algorithm AEDS. We tested different inputs like strings and files on theses algorithms (AES, DES and AEDS) on three different Machines. We have derived new Algorithm AEDS for data security and found it more robust and secure. Our AEDS Algorithm is best option over two individual Algorithms as it over comes the drawbacks of each. Brute force attack is nearly reduced by great extent as compare to rest two algorithms. Also, the time shown on the analysis (Fig.2 and Fig.3) are average time because the time may vary depend upon the processor availability and processor speed. Encryption and Decryption time for AEDS is more than that of individuals as it uses the feistel structure incorporated with DES and AES. The proposed AEDS Algorithm performs better. 


\section{International Advanced Research Journal in Science, Engineering and Technology}

Vol. 6, Issue 10, October 2019

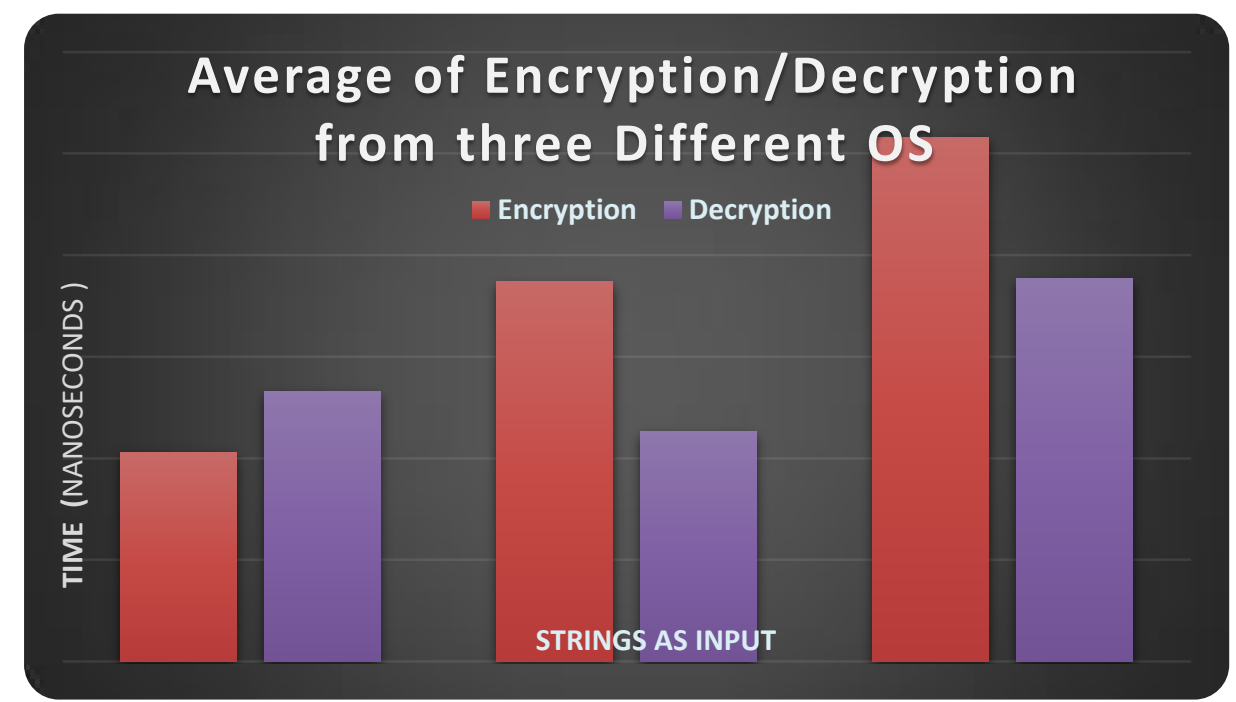

Fig.2

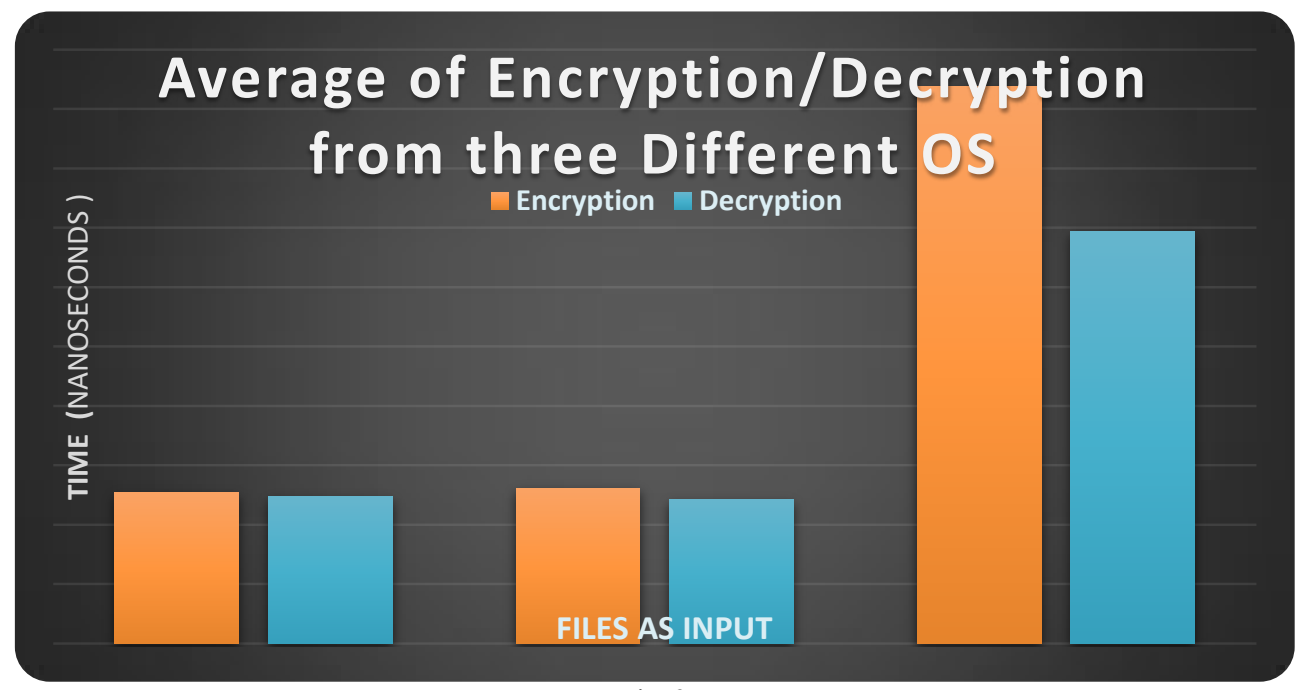

Fig.3

\section{ACKNOWLEDGMENT}

We would like to thank College of Computer Science \& Information Technology and deanship of scientific Research, Jazan University, Jazan, KSA for their support and encouragement. The author would like to thank to all authors that are listed below in the reference lists as well as anonymous reviewers for their valuable comments and suggestions that improved the presentation of this paper.

\section{REFERENCES}

[1]. Shashi Mehrotra Seth, Rajan Mishra "Comparative Analysis of Encryption Algorithms For Data Communication" IJCST Vol. 2, Issue 2, June 2011 I S N: 29 - 43 (Print)| I S S N: 0976 - 8491 (Online) www. i j c s t. c o m

[2]. DiaasalamaAbdElminaam, HatemMohamadAbdualKader,Mohly Mohamed Hadhoud, —Evolution The Performance of Symmetric Encryption Algorithms\|, international journal of network Security, vol.10,No.3,pp,216-222,May 2010

[3]. Hamdan.O.Alanazi, B.B.Zaidan, A.A.Zaidan, Hamid A.Jalab, M.Shabbir and Y. Al-Nabhani "New Comparative Study between DES, 3DES and AES within Nine Factors" Journal Of Computing, Volume 2, Issue 3, March2010, Issn2151-9617

[4]. B. Padmavathi1, S. Ranjitha Kumari2 -A Survey on Performance Analysis of DES, AES and RSA Algorithm along with LSB Substitution Technique\| International Journal of Science and Research (IJSR), India Online ISSN: 2319-7064Volume 2 Issue 4, April 2013 www.ijsr.net

[5]. AL.Jeeva, Dr.V.Palanisamy, K.Kanagaram" Comparative Analysis Of Performance Efficiency And Security Measures Of Some Encryption Algorithms "International Journal of Engineering Research and Applications (IJERA) ISSN: 2248-9622 www.ijera.com 